KUNS-1838

\title{
Twisted Moduli Stabilization in Type I String Models
}

\author{
Tetsutaro Higaki $^{1}$ and Tatsuo Kobayashi ${ }^{2}$ \\ Department of Physics, Kyoto University, Kyoto 606-8502, Japan
}

\begin{abstract}
We consider the model with the dilaton and twisted moduli fields, which is inspired by type I string models. Stabilization of their vacuum expectation values is studied. We find the stabilization of the twisted moduli field has different aspects from the dilaton stabilization.
\end{abstract}

\footnotetext{
${ }^{1}$ E-mail address: tetsu@gauge.scphys.kyoto-u.ac.jp

${ }^{2}$ E-mail address: kobayash@gauge.scphys.kyoto-u.ac.jp
} 


\section{Introduction}

Superstring theory is the promising candidate for unified theory including gravity. It has no free parameter and gauge couplings, Yukawa couplings and others of low energy effective field theory are determined by vacuum expectation values (VEVs) of dilaton/moduli fields. Thus, it is important to stabilize these VEVs. Indeed, several stabilization mechanisms have been proposed.

The dilaton/moduli fields have no potential perturbatively. Only nonperturbative effects lift their potential. Gaugino condensations are nonperturbative effects, which are plausible to generate a non-perturbative superpotential of dilaton/moduli fields. However, one can not stabilize the VEV of the dilaton field to a fine value in the model with a superpotential generated by a single gaugino condensation and the tree-level Kähler potential. One of the simple extensions is the model with double gaugino condensations and the tree-level Kähler potential, i.e. the so-called racetrack model [1]-[6], while non-perturbative Kähler potential has also been considered $[7,8,9]$. In fact, one can stabilize the VEV of the dilaton field to a finite value depending on beta function coefficients of gauge couplings relevant to gaugino condensations.

Twisted moduli fields appear in orbifold/orientifold models. These are localized at fixed points. In type I models, twisted moduli fields are gauge singlets, while they are charged in heterotic models. Gauge kinetic functions depend on twisted moduli in type I models $[10,11]$. They play a role in 4D Green-Schwarz anomaly cancellation e.g. for anomalous $U(1)[12,13]$, while the dilaton field plays the same role in heterotic models [14]. ${ }^{3}$ Thus, their VEVs determine the magnitude of Fayet-Iliopoulos terms. The prediction of the gauge couplings depends on the VEV of twisted moduli fields. The mirage unification of gauge couplings is one possibility to explain the experimental values of gauge couplings with lower string scale [16]. Hence, the magnitude of twisted moduli field VEVs is phenomenologically important.

In this paper, we will consider the model with dilaton and twisted moduli fields, which is inspired by type I string models, and study stabilization of dilaton and twisted moduli fields. For similar purpose, models with twisted moduli fields have been studied in Refs. [17, 18]. The Kähler potential of

\footnotetext{
${ }^{3}$ See also Ref.[15] for anomalous $U(1)$ in heterotic models.
} 
the twisted moduli fields is not clear. Here we will use the assumption of the canonical form, which has been studied in Ref. [19] $]^{4}$ and show this form is important to stabilize the VEV of twisted moduli. As another example, we will assume the logarithmic form of the Kähler potential for the twisted moduli fields like the dilaton and other moduli fields. That is an example of Kähler potentials, which have a different behavior from the canonical form. However, we will show that even in the case with the logarithmic Kähler potential the positive exponent in the non-perturbative superpotential is useful for the stabilization of the twisted moduli fields.

This paper is organized as follows. In the next section, we review shortly the stabilization to the dilaton VEV in the racetrack model. In section 3, we study the model with dilaton and twisted moduli fields. In section 3.1 we shortly review about the twisted moduli fields. In section 3.2 we consider the single gaugino condensation model and show how different the stabilization of twisted moduli fields is from the dilaton stabilization. In section 3.3 we consider a specific double gaugino condensation model in order to study the simultaneous stabilization of the dilaton and twisted moduli fields. In section 3.4 we give a comment about effects of twisted moduli fields on the dilaton VEV. Seciton 4 is devoted to conclusion and discussions.

\section{The racetrack model}

The tree-level Kähler potential of the dilaton field is obtained as

$$
K=-\ln (S+\bar{S})
$$

The gauge kinetic function of heterotic models is obtained as

$$
f=S
$$

up to Kac-Moody level, and the gauge coupling $g$ is obtained as $\operatorname{Re}(S)=$ $1 / g^{2}$. This is the same for the gauge multiplets originated from $D 9$ branes in type I models. Perturbatively, the dilaton field has a flat potential. The single gaugino condensation induces the non-perturbative superpotential,

$$
W=d e^{-\Delta S},
$$

\footnotetext{
${ }^{4}$ See also Ref. [20].
} 
where $d$ is a constant, $\Delta=-24 \pi^{2} / b$ and $b$ is the one-loop beta function coefficient, e.g. $b=-3 N_{c}$ for pure $\mathrm{N}=1 S U\left(N_{c}\right)$ Yang-Milles theory. With the above Kähler potential, the scalar potential $V$ is written as

$$
V=\frac{1}{S+\bar{S}}\left[\left|(S+\bar{S}) W_{S}-W\right|^{2}-3|W|^{2}\right],
$$

where $W_{S}$ denotes the first derivative of $W$ by $S$, i.e. $W_{S}=\frac{\partial W}{\partial S}$. Here we have not taken into account $D$-terms, although $S$ has a $D$-term potential in heterotic models if the model has anomalous $U(1) .{ }^{5}$ We have the following solutions of $\partial V / \partial S=0$ :

$$
(S+\bar{S}) W_{S}-W=0
$$

or

$$
(S+\bar{S})^{2} W_{S S}=2 \bar{W} \frac{(S+\bar{S}) W_{S}-W}{(S+\bar{S}) \bar{W}_{S}-\bar{W}} .
$$

With the single gaugino condensation superpotential (3), the solution (5) leads to $S+\bar{S}=-\frac{1}{\Delta}$, which is not a realistic VEV for $S$ in the asymptotically free case. The solution (6) leads to $\Delta(S+\bar{S})=\sqrt{2}$, but this corresponds to the maximum point of $V$. See Fig. 1, where the lower line shows the scalar potential against $s \equiv S+\bar{S}$ in the case with $\Delta=10$ and $d=1$.

In heterotic models, the requirement of $S L(2, Z)$ duality invariance of the overall moduli field $T$ leads to the following superpotential [21, 22, 23],

$$
W=d e^{-\Delta S} \hat{W}(T) .
$$

The corresponding scalar potential is written as

$$
V=\frac{\left|e^{-\Delta S}\right|^{2}|\hat{W}(T)|^{2}}{(S+\bar{S})(T+\bar{T})^{3}}\left[((S+\bar{S}) \Delta+1)^{2}+g(T, \bar{T})\right]
$$

with

$$
g(T, \bar{T}) \equiv \frac{1}{3}\left|(T+\bar{T}) \frac{W_{T}}{W}-3\right|^{2}-3 .
$$

Here we have used the Kähler potential of $T$ as

$$
-3 \ln (T+\bar{T}) \text {. }
$$

\footnotetext{
${ }^{5}$ In this case, the dilaton field $S$ is relevant to Green-Schwarz anomaly cancellation.
} 


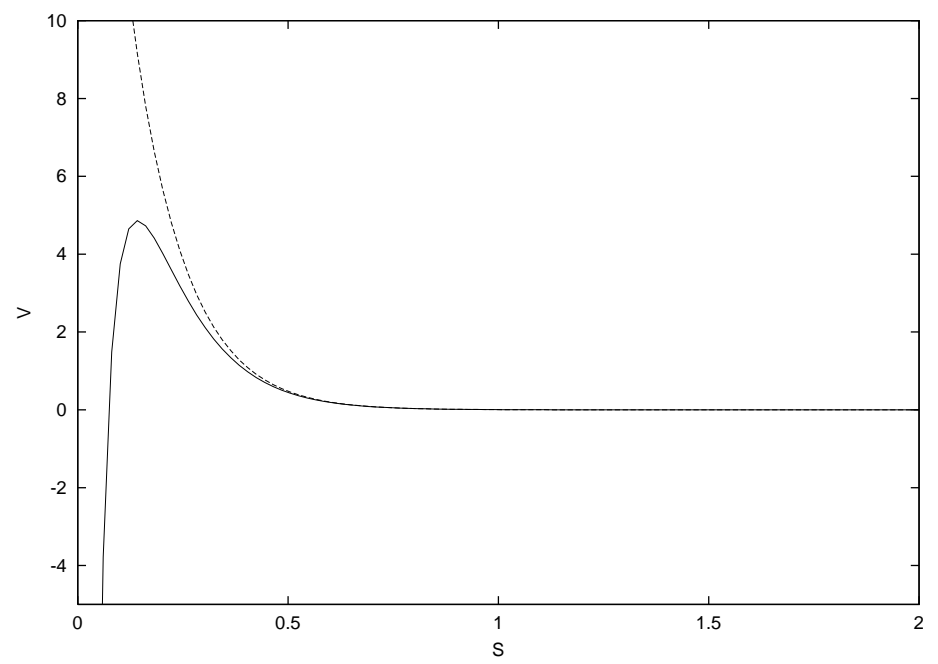

Figure 1: The lower line corresponds to the scalar potential for $\Delta=10$ and $d=1$ without $\hat{W}(T)$. The upper line corresponds to $(T+\bar{T})^{3} V /|\hat{W}(T)|^{2}$ for $\Delta=10, d=1$ and $g(T, \bar{T})=-0.5$.

However, the inclusion of $\hat{W}(T)$ does not help the stabilization of $S$. If $g(T, \hat{T})<-1$, the situation is the same as the case without $\hat{W}(T)$. If $g(T, \hat{T})>-1$, the scalar potential monotonically decreases as $s$. The upper line in Fig. 1 shows $(T+\bar{T})^{3} V /|\hat{W}(T)|^{2}$ for $\Delta=10, d=1$ and $g(T, \bar{T})=-0.5$.

One mechanism to stabilize the VEV of $S$ is to consider the superpotential with double gaugino condensations,

$$
W=d_{1} e^{-\Delta_{1} S}+d_{2} e^{-\Delta_{2} S} .
$$

With this superpotential, the solution (5) of $\partial V / \partial S=0$ is given as

$$
\begin{aligned}
\operatorname{Im}(S) & =\frac{\pi}{\Delta_{1}-\Delta_{2}}(2 n+1) \\
\operatorname{Re}(S) & =\frac{1}{\Delta_{1}-\Delta_{2}} \ln \frac{\left(1+2 \Delta_{1} \operatorname{Re}(S)\right) d_{1}}{\left(1+2 \Delta_{2} \operatorname{Re}(S)\right) d_{2}}
\end{aligned}
$$

If $\Delta_{a} \operatorname{Re}(S) \gg 1$, the latter equation becomes the simple equation,

$$
R e(S)=\frac{1}{\Delta_{1}-\Delta_{2}} \ln \frac{\Delta_{1} d_{1}}{\Delta_{2} d_{2}} .
$$


In the case with $\ln \frac{\Delta_{1} d_{1}}{\Delta_{2} d_{2}}=O(1)$, the stabilized value of $\operatorname{Re}(S)$ is determined by $1 /\left(\Delta_{1}-\Delta_{2}\right)$. Thus, the natural order of $S$ is of $O(1 / \Delta)$. If $\Delta_{1}$ and $\Delta_{2}$ are close each other, the VEV of $S$ is enhanced. For example, one can obtain $O(1)$ of $S$ in two case; i) the case with large beta-function coefficients ${ }^{6}$ $b_{i}=O(100)$ and ii) the case with fine-tuning values $\left(\Delta_{1}, \Delta_{2}\right)$. For the latter case, we have $\operatorname{Re}(S)=1 \mathrm{e}$.g. for $\left(\Delta_{2}-\Delta_{1}\right) / \Delta_{1}=0.04$ and $b_{1}=10$.

\section{Stabilization in model with twisted moduli}

\subsection{Twisted moduli}

Twisted moduli fields $M$ are localized at orbifold fixed points and these moduli fields are important from several phenomenological view points in $4 \mathrm{D}$ models obtained from type I and type II orientifold models. For example, the gauge kinetic functions corresponding to gauge groups originated from D9 branes are written as

$$
f_{a}=S+\sigma_{a} M,
$$

where $\sigma_{a}$ is a model-dependent constant $[12,25]$. Concerned about $\sigma_{a}$, here we take purely phenomenological standpoint, that is, we treat $\sigma_{a}$ as free parameters. Similarly, for the gauge groups originated from e.g. $D 5$-branes, which are wrapped on the $i$-th torus $(i=1,2,3)$, the corresponding gauge kinetic functions are written as

$$
f_{5 a}=T_{i}+\sigma_{5 a} M,
$$

where $T_{i}$ is the moduli field corresponding to the $i$-th torus and its Kähler potential is obtained

$$
K\left(T_{i}, \bar{T}_{i}\right)=-\ln \left(T_{i}+\bar{T}_{i}\right),
$$

that is, its form is exactly the same as the Kähler potential of the dilaton field (1). Thus, we can discuss the stabilization of $T_{i}$ due to gaugino condensation from $D 5$-originated gauge groups in the same way as the stabilization of $S$ due to condensation from $D 9$ gaugino fields. Here, we concentrate ourselves on the $S$ stabilization.

One of important aspects is that the twisted moduli field $M$ plays a role in 4D Green-Schwarz anomaly cancellation mechanism. For example,

\footnotetext{
${ }^{6}$ In Ref. [24] large beta-function coefficients are studied from the viewpoint of F-theory.
} 
under anomalous $U(1)$ symmetry, the twisted moduli fields are assumed to transform at one-loop level

$$
M \rightarrow M+i \delta_{G S} \Lambda
$$

with the transformation parameter $\Lambda$. The Fayet-Iliopoulos term is written by the first derivative of Kähler potential $\frac{\partial K(M, \bar{M})}{\partial M}$, where $K(M, \bar{M})$ is the Kähler potential of the twisted moduli field. Thus, the magnitude of the Fayet-Iliopoulos term is determined by the VEV of $M$.

Unfortunately, the Kähler potential of $M, K(M, \bar{M})$, is still unclear. In the limit $M \rightarrow 0$, the Kähler metric has no singularity. Hence, the Kähler potential $K(M, \bar{M})$ could be expanded as

$$
K(M, \bar{M})=\frac{1}{2}(M+\bar{M})^{2}+\cdots .
$$

Actually, this form has been studied in Ref. [19]. Thus, we use the assumption of the Kähler potential as $K(M, \bar{M})=\frac{1}{2}(M+\bar{M})^{2}$ in a half part of our analyses. However, since its reliability for $M=O(1)$ may be unclear, we assume $^{7}$

$$
K(M, \bar{M})=-\ln (M+\bar{M}),
$$

as a trial form of the Kähler potential for $M \geq O(1)$.

We also give comments on the gauge coupling unification. Within the framework of minimal supersymmetric standard model (MSSM), three gauge couplings of $S U(3) \times S U(2) \times U(1)_{Y}$ meet around $M_{X}=2 \times 10^{16} \mathrm{GeV}$. Suppose that the three gauge groups are originated from different sets of $D 9$-branes. If one can stabilize $\operatorname{Re}(S) \gg \sigma_{a} \operatorname{Re}(M)$, the gauge couplings are universal at the string scale $M_{s}$. That implies $M_{s} \approx M_{X}$. Otherwise, if $\sigma_{a} \operatorname{Re}(M)$ is sizable, the gauge couplings are, in general, non-universal at $M_{s}$. However, one of interesting possibilities to explain the experimental values of gauge couplings is the so-called "mirage unification" [16]. The MSSM gauge coupling at $\mu$ is obtained as

$$
\frac{1}{g_{a}^{2}(\mu)}=S+\sigma_{a}^{M S S M} M+\frac{b_{a}^{M S S M}}{16 \pi^{2}} \ln \frac{M_{s}^{2}}{\mu^{2}},
$$

where $b_{a}^{M S S M}$ are the one-loop beta-function coefficient for the MSSM. Let us consider a specific model that the constants $\sigma_{a}^{M S S M}$ are proportional to

\footnotetext{
${ }^{7}$ We would like to thank Kiwoon Choi for suggesting this point.
} 
$b_{a}^{M S S M}$. In this scenario, the gauge couplings are non-universal at $M_{s}$, but its prediction is the same as the universal gauge coupling around $M_{X}$. The string scale $M_{s}$ can be low depending on $\sigma_{a}^{M S S M} M$. Note that even a small value of $\operatorname{Re}(M)$ like $\sigma_{a}^{M S S M} M=O(0.01)$ is important. If the ratio of $M_{s}$ to $M_{X}$ satisfies

$$
\log _{10} \frac{M_{s}}{M_{X}} \sim \frac{\sigma_{a}^{M S S M} \operatorname{Re}(M)}{0.03},
$$

that leads to the MSSM gauge couplings consistent with the experimental values.

Thus, it is important to study the stabilization of the twisted moduli field $M$. That is the issue we will study in the flowing sections. We will also discuss how the twisted moduli field $M$ affects on the stabilization of the dilaton field $S$.

\subsection{Single gaugino condensation}

Here we study the case with single gaugino condensation, although one can not stabilize the dilaton field with the single gaugino condensation as seen section 2. That will be useful for later discussions. The Kähler potential is written as

$$
K=-\ln (S+\bar{S})+K(M, \bar{M}),
$$

and the superpotential due to the gaugino condensation is obtained as

$$
W=d e^{-\Delta(S+\sigma M)} .
$$

Using the Kähler potential and the superpotential, we can write the scalar potential as

$$
\begin{aligned}
V & =\frac{e^{K(M, \bar{M})}}{S+\bar{S}}\left[\left(K^{-1}\right)_{\bar{M}}^{M}\left|\frac{\partial K(M, \bar{M})}{\partial M} W-W_{M}\right|^{2}\right. \\
& \left.+\left|(S+\bar{S}) W_{S}-W\right|^{2}-3|W|^{2}\right]
\end{aligned}
$$

where $\left(K^{-1}\right)_{\bar{M}}^{M}$ denotes the inverse of the Kähler metric for $M$ and $\bar{M}$. Again, we do not take into account $D$-terms. Inclusion of $D$-terms would be studied elsewhere. For this scalar potential, one of solutions to the stationary condition $\frac{\partial V}{\partial M}=0$ is

$$
\left(\frac{\partial K(M, \bar{M})}{\partial M}-\Delta \sigma\right) W=0
$$


that is, $\frac{\partial K(M, \bar{M})}{\partial M}=\Delta \sigma$ is one solution.

\subsubsection{The case with $K=\frac{1}{2}(M+\bar{M})^{2}$}

To be concrete, we use the assumption of the Kähler potential $K=\frac{1}{2}(M+$ $\bar{M})^{2}$. In this case, it is convenient to define $m$ as

$$
m \equiv M+\bar{M}-\Delta \sigma .
$$

Then the scalar potential is written as

$$
V=\frac{e^{-\Delta(S+\bar{S})-\sigma^{2} \Delta^{2} / 2}}{S+\bar{S}} e^{m^{2} / 2}\left[m^{2}+g(S+\bar{S})\right],
$$

where

$$
g(S+\bar{S}) \equiv\left|(S+\bar{S}) \frac{W_{S}}{W}-1\right|^{2}-3
$$

For the single gaugino condensation, we have

$$
g(S+\bar{S})=(\Delta(S+\bar{S})+1)^{2}-3 .
$$

The solutions of the stationary condition $\frac{\partial V}{\partial m}=0$ are obtained as follows,

$$
m=0, \quad m= \pm \sqrt{-2-g(S+\bar{S})} .
$$

The former solution corresponds to Eq.(26). The latter solutions are allowed only if

$$
2+g(S+\bar{S})<0
$$

By the definition (30), this inequality is never satisfied for $(S+\bar{S})>0$. We have $\frac{\partial^{2} V}{\partial m^{2}}>0$ for the former solution $m=0$ if

$$
2+g(S+\bar{S})>0
$$

By the definition (30), this inequality is always satisfied for $(S+\bar{S})>0$. In addition, for the latter solution, we always have $\frac{\partial^{2} V}{\partial m^{2}}>0$ if the solution is realized, i.e. $2+g(S+\bar{S})<0$. In Fig. 2 the upper and lower lines show $v \equiv e^{m^{2} / 2}\left[m^{2}+g(S+\bar{S})\right]$ for $g(S+\bar{S})=-1$ and -3 , respectively.

More explicitly, these solutions lead to the following values of $\operatorname{Re}(M)$,

$$
2 R e(M)=-\Delta \sigma, \quad 2 R e(M)=-\Delta \sigma \pm \sqrt{-2-g(S+\bar{S})} .
$$




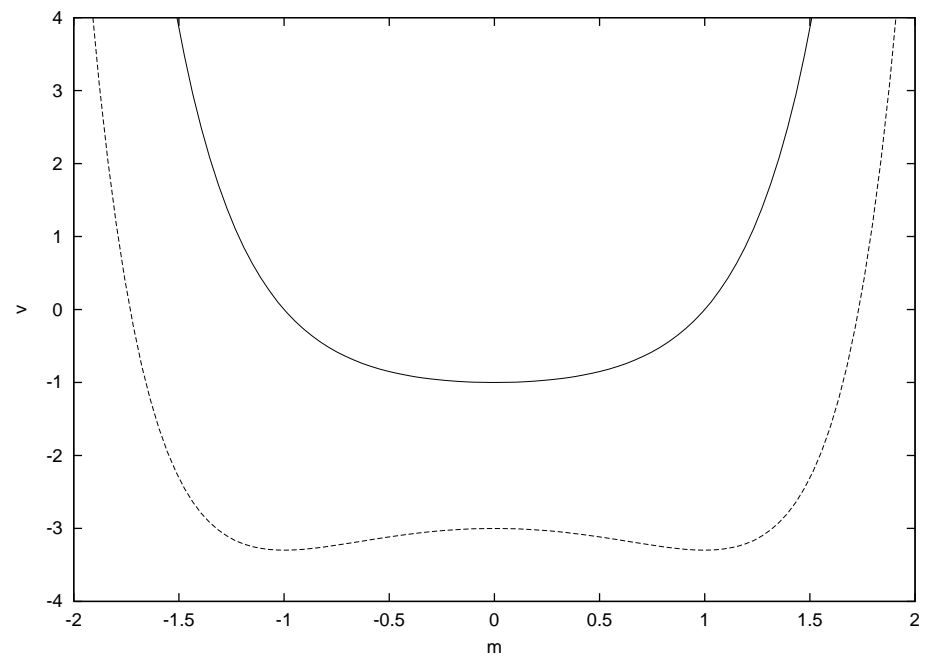

Figure 2: The upper and lower lines show $v \equiv e^{m^{2} / 2}\left[m^{2}+g(S+\bar{S})\right]$ for $g(S+\bar{S})=-1$ and -3 , respectively.

In the case that the Kähler potential $K(M, \bar{M})=\frac{1}{2}(M+\bar{M})^{2}$ is reliable, in particular $\operatorname{Re}(M)<O(1)$, these results are valid. Similar analyses can be done for the polynomial Kähler potential. However, it is not clear that the expansion of the Kähler potential $K(M, \bar{M})=\frac{1}{2}\left(M+\bar{M}^{2}\right)+\cdots$ is reliable for $\operatorname{Re}(M) \geq O(1)$. Thus, in the next subsection we will perform the same analysis by assuming $K=-\ln (M+\bar{M})$ as a trial. That is an example of Kähler potentials which have the behavior opposite to the canonical form at large $M$.

\subsubsection{The case with $K=-\ln (M+\bar{M})$}

Here the same analysis as subsection 3.2.1 will be done with the assumption $K=-\ln (M+\bar{M})$. In this case, it is convenient to define

$$
m^{\prime} \equiv(M+\bar{M}) \sigma \Delta+1 \text {. }
$$

Using this variable, we can write the scalar potential (25)

$$
V=\frac{\sigma \Delta}{(S+\bar{S})\left(m^{\prime}-1\right)} e^{-\Delta(S+\bar{S})+2} e^{-m^{\prime}}\left(m^{\prime 2}+g(S+\bar{S})\right) \text {. }
$$


The solutions of the stationary condition $\frac{\partial V}{\partial m^{\prime}}=0$ are obtained

$$
m^{\prime}=0, \quad m^{\prime}=1 \pm \sqrt{-g(S+\bar{S})-1} .
$$

The latter solution is allowed only if

$$
g(S+\bar{S})<-1
$$

For $\sigma<0$, the region with $\operatorname{Re}(M)>0$ corresponds to $m^{\prime}<1$. In this case, the second derivative of the scalar potential, $\frac{\partial^{2} V}{\partial m^{\prime 2}}$ is positive at $m^{\prime}=0$ if

$$
g(S+\bar{S})>-2 .
$$

This is always satisfied by the definition $(30)$ if $(S+\bar{S})>0$. At $m^{\prime}=$ $1-\sqrt{-g(S+\bar{S})-1}$, we have $\frac{\partial^{2} V}{\partial m^{\prime 2}}>0$ if

$$
g(S+\bar{S})<-2
$$

This is never satisfied by the definition $(30)$ if $(S+\bar{S})>0$. Fig. 3 shows $v=-\frac{e^{-m^{\prime}}}{m^{\prime}-1}\left[m^{\prime 2}+g(S+\bar{S})\right]$ for $g(S+\bar{S})=-1.5$ and -3 , respectively. The scalar potential has a singularity at $m^{\prime}=1$, which comes from the singularity of the Kähler potential at $M=0$. However, at the vicinity of $M=0$ the Kähler potential $K(M, \bar{M})=\frac{1}{2}(M+\bar{M})^{2}$ as studied in the previous subsection is rather reliable than the Kähler potential $-\ln (M+\bar{M})$.

For $\sigma>0$, the region with $\operatorname{Re}(M)>0$ corresponds to $m^{\prime}>1$. However, the second derivative of the scalar potential, $\frac{\partial^{2} V}{\partial m^{\prime 2}}$ is always negative at $m^{\prime}=$ $1+\sqrt{-g(S+\bar{S})-1}$. This situation is the same as the problem of the dilaton stabilization by the single gaugino condensation as seen in Section 2 .

Thus, the model with $\sigma<0$ is interesting for $K(M, \bar{M})=-\ln (M+\bar{M})$, that is, the positive exponent of $M$ in the superpotential is useful. The solutions $m^{\prime}=0$ and $1-\sqrt{-g(S+\bar{S})-1}$ corresponds to

$$
R e(M)=\frac{-1}{\sigma \Delta}, \quad \frac{-\sqrt{-g(S+\bar{S})-1}}{\sigma \Delta},
$$

respectively.

Assuming the Kähler potential $K(M, \bar{M})=\frac{1}{2}(M+\bar{M})^{2}$ and $-\ln (M+\bar{M})$, we have shown that the VEV of $\operatorname{Re}(M)$ can be stabilized with the VEV 


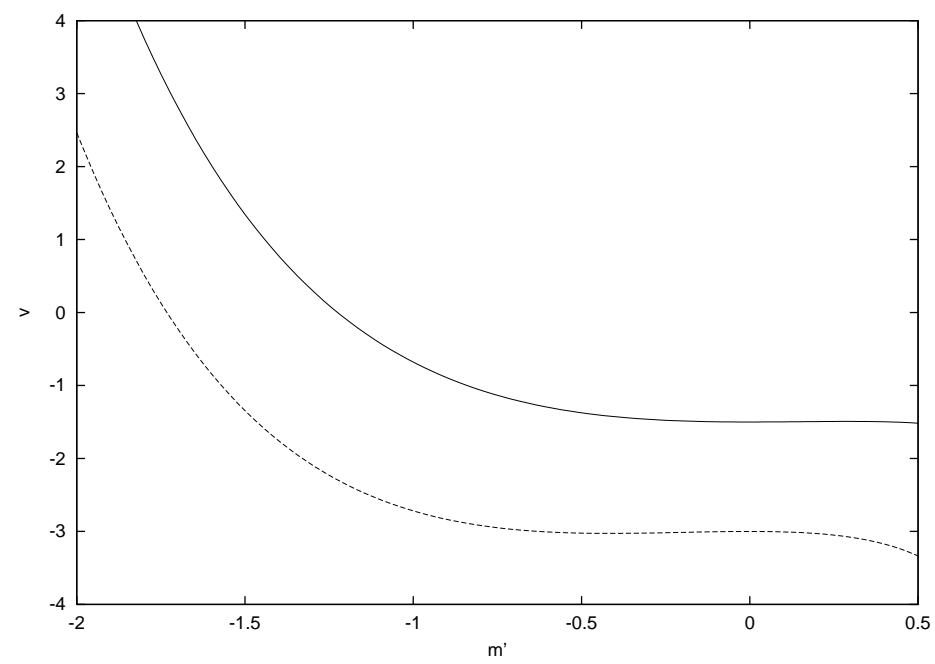

Figure 3: The upper and lower lines show $v=-\frac{e^{-m^{\prime}}}{m^{\prime}-1}\left[m^{\prime 2}+g(S+\bar{S})\right]$ for $g(S+\bar{S})=-1.5$ and -3 , respectively.

of $S$ fixed. The former case implies that the canonical Kähler potential is important for the stabilization of the twisted moduli. Such analysis can be extended into the case with polynomial Kähler potential $K(M, \bar{M})$. On the other hand, the latter case with $K(M, \bar{M})=-\ln (M+\bar{M})$ shows that even with the logarithmic Kähler potential, the positive exponent in the nonperturbative superpotential is useful to stabilize the VEV of twisted moduli fields. It is speculative whether really $K(M, \bar{M})=-\ln (M+\bar{M})$ for large $M$, but that is an example of Kähler potentials which have the behavior opposite to the canonical form at large $M$. For other forms of Kähler potential, the analysis can be extended. The key-point in the stabilization of twisted moduli is that the polynomial form of Kähler potential is useful and the positive exponent of $M$ in the superpotential is helpful. These aspects differ from the dilaton stabilization. The positive exponent of the dilaton field in the non-perturbative superpotential corresponds to the asymptotically non-free case $^{8}$.

Of course, the VEV of $S$ is not stabilized in the case with the single gaugino condensation which discussed in this subsection. In order to study the stabilization of $S$ and $M$ at the same time, we will consider the double

\footnotetext{
${ }^{8}$ See Ref. [26] for the dilaton stabilization in the asymptotically non-free case.
} 
gaugino condensations in the following subsections.

\subsection{Mirage model}

Here we consider the superpotential generated from the double gaugino condensations, i.e., the racetrack model,

$$
W=d_{1} e^{-\Delta_{1}\left(S+\sigma_{1} M\right)}+d_{2} e^{-\Delta_{2}\left(S+\sigma_{2} M\right)} .
$$

The mirage unification can occur in the case that $\sigma_{a}^{M S S M}$ for the MSSM are proportional to the one-loop beta-function coefficients $b_{a}^{M S S M}$. Here we consider a specific type of gaugino condensation models that $\sigma_{a}$ for the double gaugino condensations are proportional to their one-loop beta-function coefficients, that is, we can write

$$
\Delta_{a} \sigma_{a}=C,
$$

where $C$ is common for the double gaugino condensations for $a=1,2$. Then, the superpotential can be written as

$$
W=e^{-C M} \tilde{W}, \quad \tilde{W}=\left(d_{1} e^{-\Delta_{1} S}+d_{2} e^{-\Delta_{2} S}\right) .
$$

The corresponding scalar potential is written as

$$
\begin{aligned}
V & =\frac{e^{K(M, \bar{M})-C(M+\bar{M})}}{S+\bar{S}}\left[\left(K^{-1}\right) \frac{M}{M}\left|\frac{\partial K(M, \bar{M})}{\partial M}-C\right|^{2}|\tilde{W}|^{2}\right. \\
& \left.+\left|(S+\bar{S}) \tilde{W}_{S}-\tilde{W}\right|^{2}-3|\tilde{W}|^{2}\right] .
\end{aligned}
$$

The racetrack solution $(13,14)$ corresponding to $(S+\bar{S}) \tilde{W}_{S}-\tilde{W}=0$ is still a

solution of $\frac{\partial V}{\partial S}=0$ for the present scalar potential. Here we restrict ourselves to this solution and the VEV of $S$ itself is obtained by Eqs. $(13,14)$. The analysis on the scalar potential for the twisted moduli is almost the same as what has been done in section 3.2. The present case corresponds to the case with $g(S+\bar{S})=-3$ and $\Delta \sigma=C$.

To be concrete, we again use the assumption of $K(M, \bar{M})$ as the canonical form and logarithmic form. First, in the case with $K(M, \bar{M})=\frac{1}{2}(M+\bar{M})^{2}$ the solutions of $\frac{\partial V}{\partial m}=0$ are obtained as

$$
R e(M)=\frac{C}{2}, \quad \frac{C \pm 1}{2} .
$$


For the former solution, we have $\frac{\partial^{2} V}{\partial m^{2}}<0$ because of $g(S+\bar{S})=-3$. If there are additional contributions increasing the value of $g(S+\bar{S})$, this solution could be a local minimum. On the other hand, for the latter solution $2 R e(M)=C \pm 1$, we have $\frac{\partial^{2} V}{\partial m^{2}}>0$ as well as $\frac{\partial^{2} V}{\partial m \partial S}>0$. At this point, the $F$-component of $M$ is obtained as

$$
\left|F_{M}\right|=\frac{1}{\sqrt{S+\bar{S}}} e^{\frac{1}{4}\left(1-C^{2}\right)}|\tilde{W}| .
$$

Similarly, we can analyse the potential minima for the assumed Kähler potential $K(M, \bar{M})=-\ln (M+\bar{M})$. We are interested in the case with $C<0$. The solutions of $\frac{\partial V}{\partial m^{\prime}}=0$ are obtained as

$$
\operatorname{Re}(M)=-\frac{1}{2 C}, \quad \pm \frac{1}{\sqrt{2} C} .
$$

For the former solution, $\operatorname{Re}(M)=-\frac{1}{2 C}$, we have $\frac{\partial^{2} V}{\partial m^{\prime 2}}<0$ because of $g(S+$ $\bar{S})=-3$. Additional contributions increasing $g(S+\bar{S})$ might make this point a local minimum. For the solution $\operatorname{Re}(M)=-\frac{1}{\sqrt{2} C}$, we have $\frac{\partial^{2} V}{\partial m^{\prime 2}}>0$ as well as $\frac{\partial^{2} V}{\partial m^{\prime} \partial S}>0$. At this point, the $F$-component of $M$ does not vanish. Furthermore, stabilized values must satisfy the constraint $\operatorname{Re}\left(f_{a}\right)=\operatorname{Re}(S)+$ $\sigma_{a} \operatorname{Re}(M)>0$. For the above solution $\operatorname{Re}(M)=-\frac{1}{\sqrt{2} C}$, we can write

$$
\operatorname{Re}\left(f_{a}\right)=\operatorname{Re}(S)-\frac{1}{\sqrt{2} \Delta_{a}} .
$$

Thus, the stabilized value of $S(14)$ must satisfy $\operatorname{Re}(S)>\frac{1}{\sqrt{2} \Delta_{a}}$. Recall that the natural order of $S$ is of $O\left(\frac{1}{\Delta}\right)$ unless $\Delta_{a}$ are close each other or $\ln \frac{\Delta_{1} d_{1}}{\Delta_{2} d_{2}}$ is large.

\subsection{Generic racetrack model}

In the previous section, we considered the specific racetrack model, i.e. $\sigma_{1} \Delta_{1}=\sigma_{2} \Delta_{2}$. For generic case $\sigma_{1} \Delta_{1} \neq \sigma_{2} \Delta_{2}$, analyses become complicated. Here we give a comment on such generic case.

As solutions of $\partial V / \partial S=0$, we again concentrate on the solution (5). That leads to the following equations,

$$
\ln \frac{\left(2 \Delta_{1} \operatorname{Re}(S)+1\right) d_{1}}{\left(2 \Delta_{2} \operatorname{Re}(S)+1\right) d_{2}}=\left(\Delta_{1}-\Delta_{2}\right) \operatorname{Re}(S)+\left(\Delta_{1} \sigma_{1}-\Delta_{2} \sigma_{2}\right) \operatorname{Re}(M),
$$




$$
\left(\Delta_{1}-\Delta_{2}\right) \operatorname{Im}(S)+\left(\Delta_{1} \sigma_{1}-\Delta_{2} \sigma_{2}\right) \operatorname{Im}(M)=(2 n+1) \pi .
$$

Furthermore, if $\Delta \operatorname{Re}(S) \gg 1$, we obtain

$$
\operatorname{Re}(S)=\frac{\left(\Delta_{1} \sigma_{1}-\Delta_{2} \sigma_{2}\right)}{\Delta_{2}-\Delta_{1}} \operatorname{Re}(M)+\frac{1}{\Delta_{1}-\Delta_{2}} \ln \frac{\Delta_{1} d_{1}}{\Delta_{2} d_{2}} .
$$

The second term in the right hand side is the same as Eq.(14). The first term is a new contribution from $M$. When $\Delta_{1} \sigma_{1}=\Delta_{2} \sigma_{2}$, the first term vanishes and that is consistent with the subsection 3.3. However, if $\Delta_{1} \sigma_{1} \neq \Delta_{2} \sigma_{2}$, the $\mathrm{VEV}$ of $\operatorname{Re}(M)$ corresponds effectively to a large difference of $d_{a}$ in Eq.(14) as seen by replacing $d_{a} \rightarrow d_{a} e^{-\Delta_{a} \sigma_{a} R e(M)}$. Thus, the value of $\operatorname{Re}(M)$ is important to the stabilized value of $\operatorname{Re}(S)$.

Suppose that the VEV of $\operatorname{Re}(M)$ is also stabilized by the following equation similar to Eq.(5)

$$
\frac{\partial K(M, \bar{M})}{\partial M} W+W_{M}=0
$$

Combined with Eq.(50), for $\Delta R e(S) \gg 1$, we obtain

$$
\frac{\partial K}{\partial M}=\frac{\Delta_{1} \Delta_{2}\left(\sigma_{1}-\sigma_{2}\right)}{\left(\Delta_{2}-\Delta_{1}\right)}
$$

For example, that leads to

$$
R e(M)=\frac{\Delta_{1} \Delta_{2}\left(\sigma_{1}-\sigma_{2}\right)}{2\left(\Delta_{2}-\Delta_{1}\right)},
$$

for $K(M, \bar{M})=\frac{1}{2}(M+\bar{M})^{2}$, and

$$
\operatorname{Re}(M)=\frac{\Delta_{2}-\Delta_{1}}{2 \Delta_{1} \Delta_{2}\left(\sigma_{1}-\sigma_{2}\right)},
$$

for $K(M, \bar{M})=-\ln (M+\bar{M})$. In the former (latter) case, the value of $\operatorname{Re}(M)$ is enhanced (suppressed) for fine-tuning $\Delta_{1} \approx \Delta_{2}$, while it is suppressed (enhanced) for fine-tuning $\sigma_{1} \approx \sigma_{2}$. Eq.(52) becomes

$$
\operatorname{Re}(S)=\left(-\sigma_{1}+\frac{2}{\Delta_{1}} \operatorname{Re}(M)\right) \operatorname{Re}(M)+\frac{1}{\Delta_{1}-\Delta_{2}} \ln \frac{\Delta_{1} d_{1}}{\Delta_{2} d_{2}}
$$


for $K(M, \bar{M})=\frac{1}{2}(M+\bar{M})^{2}$, and

$$
\operatorname{Re}(S)=-\sigma_{1} \operatorname{Re}(M)+\frac{1}{2 \Delta_{1}}+\frac{1}{\Delta_{1}-\Delta_{2}} \ln \frac{\Delta_{1} d_{1}}{\Delta_{2} d_{2}}
$$

for $K(M, \bar{M})=-\ln (M+\bar{M})$. For the latter case, the first term in the right hand side would be important when $\operatorname{Re}(M)$ is enhanced by fine-tuning

$\sigma_{1} \approx \sigma_{2}$. Thus, the value of $\operatorname{Re}(M)$ has the interesting effect on the stabilized value of $\operatorname{Re}(S)$.

\section{Conclusion}

We have studied stabilization of the dilaton and twisted moduli by assuming the canonical and logarithmic forms for the Kähler potential of the twisted moduli field. The canonical Kähler potential plays a role in the stabilization of the twisted moduli. This analysis can be extended into the case with polynomial Kähler potential. On the other hand, even with the logarithmic Kähler potential, the positive exponent of the twisted moduli field in the nonperturbative superpotential is significant. The logarithmic form was used as an example of Kähler potentials which have different behavior from the canonical form. That suggests that even for such case the positive exponent of the twisted moduli fields in the superpotentail would be helpful. These aspects are different from the dilaton stabilization.

Simialrly, in the models that gauge kinetic functions depend linearly on two or more moduli fields, the positive exponent of those fields in the superpotential might be helpful for the moduli stabilization.

Also we have considered the specific racetrack model with $\sigma_{1} \Delta_{1}=\sigma_{2} \Delta_{2}$ in order to discuss stabilization of the dilaton and twisted moduli at the same time. In generic case, the VEV of $M$ affects the stabilized value of the dilaton VEV. This point is also important in the stabilization of the twisted moduli fields.

The knowledge on the Kähler potential of the twisted moduli field is necessary to investigate numerically reliable results. We have not taken into account $D$-terms. Inclusion of $D$-terms would be studied elsewhere.

The models have been studied lead to the negative cosmological constant. That is a common problem as the dilaton stabilization. The vanishing 
cosmological constant could be realized by the models with more gaugino condensations [5], non-perturbative Kähler potential [9] or R-symmetry [27].

\section{Acknowledgment}

The authors would like to thank Kiwoon Choi, Yoshiharu Kawamura and Hiroaki Nakano for useful discussions. T. K. is supported in part by the Grant-in-Aid for Scientific Research from Ministry of Education, Science, Sports and Culture of Japan (\#14540256).

\section{References}

[1] N. V. Krasnikov, Phys. Lett. B 193, 37 (1987).

[2] L. J. Dixon, SLAC-PUB-5229 Invited talk given at 15th APS Div. of Particles and Fields General Mtg., Houston, TX, Jan 3-6, 1990

[3] T. R. Taylor, Phys. Lett. B 252, 59 (1990).

[4] J. A. Casas, Z. Lalak, C. Munoz and G. G. Ross, Nucl. Phys. B 347, 243 (1990).

[5] B. de Carlos, J. A. Casas and C. Munoz, Nucl. Phys. B 399, 623 (1993).

[6] M. Dine and Y. Shirman, Phys. Rev. D 63, 046005 (2001).

[7] T. Banks and M. Dine, Phys. Rev. D 50, 7454 (1994).

[8] P. Binetruy, M. K. Gaillard and Y. Y. Wu, Nucl. Phys. B 481, 109 (1996).

[9] J. A. Casas, Phys. Lett. B 384, 103 (1996).

[10] G. Aldazabal, A. Font, L. E. Ibanez and G. Violero, Nucl. Phys. B 536, 29 (1998).

[11] See for effective low-energy Lagrangian of Type I models L. E. Ibanez, C. Munoz and S. Rigolin, Nucl. Phys. B 553, 43 (1999) and references therein. 
[12] L. E. Ibanez, R. Rabadan and A. M. Uranga, Nucl. Phys. B 542, 112 (1999).

[13] Z. Lalak, S. Lavignac and H. P. Nilles, Nucl. Phys. B 559, 48 (1999).

[14] E. Witten, Phys. Lett. 149B (1984) 351;

M. Dine, N. Seiberg and E. Witten, Nucl. Phys. B289 (1987) 589;

W. Lerche, B.E.W. Nilsson and A.N. Schellekens, Nucl. Phys. B289 (1987) 609 .

[15] T. Kobayashi and H. Nakano, Nucl. Phys. B496 (1997) 103;

G.B. Cleaver and A.E. Faraggi, Int. J. Mod. Phys. A14 (1999) 2335.

[16] L. E. Ibanez, arXiv:hep-ph/9905349.

[17] S. A. Abel and G. Servant, Nucl. Phys. B 597, 3 (2001).

[18] R. Ciesielski and Z. Lalak, JHEP 0212, 028 (2002).

[19] E. Poppitz, Nucl. Phys. B 542, 31 (1999).

[20] C. A. Scrucca and M. Serone, JHEP 0007, 025 (2000).

[21] S. Ferrara, D. Lust, A. D. Shapere and S. Theisen, Phys. Lett. B 225, 363 (1989).

[22] S. Ferrara, N. Magnoli, T. R. Taylor and G. Veneziano, Phys. Lett. B 245, 409 (1990).

[23] A. Font, L. E. Ibanez, D. Lust and F. Quevedo, Phys. Lett. B 245, 401 (1990).

[24] V. Kaplunovsky and J. Louis, Phys. Lett. B 417, 45 (1998).

[25] I. Antoniadis, C. Bachas and E. Dudas, Nucl. Phys. B 560, 93 (1999).

[26] C. P. Burgess, A. de la Macorra, I. Maksymyk and F. Quevedo, Phys. Lett. B 410, 181 (1997).

[27] K. I. Izawa and T. Yanagida, Prog. Theor. Phys. 101, 171 (1999). 